\title{
Analyse de la diversité génétique de la race bovine Bororo (Wodaabé) du Niger à l'aide de marqueurs microsatellites
}

\author{
Mahaman Maaouia Abdou MOUSSA ${ }^{1}$, Moustapha GREMA ${ }^{2,3}$, \\ Stéphane A.R. TAPSOBA ${ }^{4}$, Moumouni ISSA ${ }^{2}$, Amadou TRAORE $^{4 *}$, \\ Marichatou HAMANI ${ }^{1}$, Rudolph PICHLER ${ }^{5}$, Albert SOUDRE ${ }^{6}$, Moumouni SANOU $^{4}$, \\ Hamidou H. TAMBOURA ${ }^{4}$, Yenikoye ALHASSANE ${ }^{1}$ et Kathiravan PERIASAMY ${ }^{5}$ \\ ${ }^{1}$ Université Abdou Moumouni de Niamey, Faculté d'Agronomie BP 10960, Niamey, Niger. \\ ${ }^{2}$ Université Abdou Moumouni de Niamey, Faculté des Sciences et Techniques BP 10662, Niamey, Niger. \\ ${ }^{3}$ Université de Diffa, Faculté des Sciences Agronomiques BP 78 Diffa, Niger. \\ ${ }^{4}$ INERA, Laboratoire de Biologie et santé animales, 04 BP 8645 Ouagadougou 04, Burkina Faso. \\ ${ }^{5}$ Animal Production and Health Laboratory, Joint FAO/IAEA Division, International Atomic Energy Agency, \\ Vienna, Austria. \\ ${ }^{6}$ Université de Koudougou, BP 376 Koudougou, Burkina Faso. \\ *Auteur correspondant ; E-mail: traore_pa@yahoo.fr ; Tél: +22670429440; Fax: +22625340272.
}

\section{RESUME}

La diversité génétique de 48 individus de la race bovine Bororo du Niger provenant de trois populations a été étudiée à l'aide d'un panel de 27 marqueurs microsatellites. Un total de 201 allèles différents a été détecté sur les 27 loci explorés. Le nombre d'allèles observés (Na) par locus varie de 2 (ETH152 et SPS115) à 10 (TGLA53) avec une moyenne de 7,4 allèles observés par locus. Les taux d'hétérozygoties observés étaient compris entre 0,25 (ETH3) et 0,94 (BM2113) avec des valeurs moyennes par population supérieures à 0,63 . En dépit d'un faible déficit en hétérozygotes observé, l'ensemble de la population évolue sous l'équilibre de Hardy-Weinberg. Cependant, la différenciation génétique entre ces trois populations est très faible avec un taux global de $\mathrm{F}_{\mathrm{ST}}$ de 0,035 indiquant que 3,5\% de la variation totale observée est liée aux différences entre populations. Cette situation est à mettre sur le compte d'un flux génique important $(\mathrm{Nm}>5,7 \%)$ entre ces trois populations en relation avec le mode de gestion des troupeaux. A long terme, ce flux de gênes important pourrait altérer la diversité génétique des zébus bororo dans ces trois localités du Niger.

(C) 2019 International Formulae Group. All rights reserved

Mots clés: Zébu Bororo, marqueurs microsatellites, diversité génétique, Niger.

\section{Molecular analysis of genetic diversity of Niger Bororo (Wodaabé) cattle breed using microsatellites markers}

\begin{abstract}
Genetic diversity of 48 Niger Bororo individuals from three populations have been assessed using a panel of 27 microsatellite markers. A total of 201 different alleles were generated on the 27 loci explored. The number of observed alleles (Na) per locus ranged from 2 (ETH152 and SPS115) to 10 (TGLA53) with an average of 7.4 observed alleles per locus. Observed heterozygosity values ranged between 0.25 (ETH3) and
\end{abstract}


0.94 (BM2113). The overall mean observed heterozygosity value was more than 0.63 . Despite a moderate heterozygote deficiency, the whole population did not deviate significantly from Hardy-Weinberg equilibrium. However, genetic differentiation between these three populations was low (3.5\%). This situation is attributable to a significant gene flow $(\mathrm{Nm}>5.7 \%)$ between populations in relation to the herd management system. In the long term, this important gene flow could alter the genetic diversity of zebu Bororo in these three areas of Niger.

(C) 2019 International Formulae Group. All rights reserved

Keywords: Bororo cattle, microsatellite markers, genetic diversity, Niger.

\section{INTRODUCTION}

Le Niger est un pays d'élevage, par excellence, à vocation essentiellement pastorale. Le secteur agricole (agriculture et élevage) participe à hauteur de 39,5\% au PIB dont $24 \%$ pour l'élevage. Le bétail se compose principalement de bovins et de petits ruminants (INS, 2008).

Au Niger, l'élevage représente pour les agro-éleveurs et les pasteurs un mode de production permettant de satisfaire leurs besoins alimentaires, mais aussi un mode de vie socio-culturel en parfaite symbiose avec le milieu physique dans lequel ils évoluent.

Le système extensif qui concerne la grande majorité des effectifs du cheptel est basé sur l'exploitation des ressources naturelles en zones agropastorale et pastorale. La disponibilité de ces ressources dépend de la pluviosité qui varie très fortement dans l'espace et dans le temps. Au regard des épisodes de sècheresses qu'a connu le Niger, des centres adaptés de sélection et de multiplication de bétails ont été construits dans chaque région du pays, pour d'une part, sauvegarder dans leur niche écologique les différentes races animales locales et, d'autre part reconstituer le cheptel décimé. Ces centres ont aussi la vocation de diffuser des animaux à haut potentiel de production dans les élevages traditionnels, de vulgariser des méthodes modernes d'élevage en zone rurale. C'est dans ce cadre que le zébu Azawak et le taurin Kouri, qui sont élevés respectivement dans les stations de Toukounous (Tillabéry) et Sayam (Diffa), ont bénéficié d'un programme de sélection phénotypique et d'activités de caractérisation. Cependant, il n'existe pas encore de centre fonctionnel de multiplication et de diffusion pour le zébu Bororo, un centre a été mis en place à Fako (Maradi) mais ce dernier n'a pas fonctionné en raison des mortalités occasionnées consécutive à la grande sècheresse de 1984 (Habou et al., 2016). Le zébu Bororo du Niger n'a donc pas été l'objet d'études ni en milieu réel, ni en station pour un suivi régulier en vue de sa caractérisation ou d'actions de sélection.

Le zébu Bororo est pourtant l'une des principales races du Niger qui doit son nom à l'une des tribus Peulhs du pays qui l'élève, les Wodaabés (Bororos) pour qui, cette race joue un rôle socio-culturel et aussi économique. En dépit de son tempérament difficile rapporté par les éleveurs des autres races (farouche, difficile à dresser, mauvaise productrice, etc.), certains peulhs bororos l'élèvent exclusivement du fait de son élégance, son attachement et sa réputation à obéir aux ordres de son maître.

Le zébu Bororo est présent au-delà des frontières nationales. Il est retrouvé dans beaucoup de pays de la sous-région (Nigeria, Bénin, Tchad, Cameroun et en République Centrafricaine), où il reçoit différentes appellations telles que M'Bororo, Rahadji, Hanagamba, zébu Peuhl rouge, Djafoun, Fellata, Foulata etc. (Zangui, 1986).

Pour beaucoup de races bovines en Afrique de l'Ouest, les données scientifiques nécessaires à la prise de décision sont fragmentaires ou absentes (Boma et al., 2018, Kassa et al., 2016). Des études de caractérisation, dans des contextes non Nigérien, ont été conduites sur cette race sur des aspects phénotypiques (Douffissa, 1993), et génotypiques au Cameroun et au Nigeria (Ibeagha-Awemu et al., 2006). Au Niger, il y a eu seulement une description qualitative sommaire (Maaouia et al., 2018) sans aborder les aspects génétiques qui doivent être utilisés pour confirmer les tendances obtenues dans 
les études phénotypiques (Boma et al., 2018). La capitalisation de ces aspects dans un schéma de sa caractérisation est pourtant fondamentale pour mieux définir des stratégies d'amélioration génétique et de valorisation du bovin zébu bororo dans son milieu écologique. La présente étude s'inscrit dans ce cadre et l'objectif est d'évaluer la diversité et d'inférer la structure génétique de la race bovine bororo du Niger en utilisant le polymorphisme de 27 marqueurs microsatellites.

\section{MATERIEL ET METHODS \\ Echantillonnage et extraction d'ADN}

L'étude a été conduite dans trois départements du Niger: Bangui, Bermo et Diffa (Figure 1). Ces sites ont été choisis car ils constituent les niches écologiques des sujets zébus bororo supposés plus typiques, homogènes, avec moins de métissage avec les autres races locales (Alhassane et al., 2018). Ces départements sont marqués par un climat de type sahélien avec trois saisons distinctes:

- Une saison sèche et froide de Novembre à février ;

- Une saison sèche et chaude de Mars à mai ;

- Une saison pluvieuse de juin à septembre, pouvant aller à la mioctobre.

La pluviométrie est comprise entre 150 et $400 \mathrm{~mm}$ par an. La végétation est à dominance d'épineux et de graminées annuelles et pérennes notamment du Panicum turgidum et Aristida adsensionis.

Le matériel biologique était constitué de sang. Les échantillons ont été prélevés sur trois sites à raison de 16 individus par site. Les sites sont la commune de Bangui $\left(13^{\circ}\right.$ $\left.400 \mathrm{~N}, 06^{\circ} 16^{\prime} \mathrm{E}\right)$ dans la région de Tahoua, le site de Bermo $\left(15^{\circ} 160 \mathrm{~N}, 06^{\circ} 440 \mathrm{E}\right)$ dans la région de Maradi et le site de Diffa (entre $12^{\circ} 20^{\prime}-14^{\circ} 20^{\prime}$ de latitude Nord et $13^{\circ}-15^{\circ} 30$ 'de longitude Est) dans la région de Diffa. Le sang a été prélevé par ponction jugulaire dans un tube EDTA.

L'ADN a été extrait du sang total en utilisant un kit d'extraction, MasterPure DNA Purification Kit (Biozym Illumina Inc, USA) selon les recommandations du fabricant.
L'ADN a été conservé à $4{ }^{\circ} \mathrm{C}$ jusqu'à l'amplification par PCR.

\section{Amplification par PCR et génotypage}

Un panel de 27 marqueurs microsatellites recommandés par la FAO (FAO, 2011) et des amorces conjuguées avec l'un des trois colorants fluorescents (FAM, HEX et ATTO550) ont été utilisés pour l'analyse de la diversité.

La PCR a été réalisée dans les conditions suivantes : dénaturation initiale pendant 5 min à $95^{\circ} \mathrm{C}$ suivie de 35 cycles de dénaturation à $95^{\circ} \mathrm{C}$ pendant $30 \mathrm{~s}, 1$ minute d'hybridation à des températures spécifiques à chaque amorce, une élongation à $72{ }^{\circ} \mathrm{C}$ pendant 1 min et une extension finale à $72{ }^{\circ} \mathrm{C}$ pour $10 \mathrm{~min}$. Les produits PCR ont ensuite été soumis à une électrophorèse après multiplexage dans un séquenceur d'ADN automatique ABI3100 (Applied Biosystems, USA) en utilisant le ROX500 (Applied Biosystems, USA) comme marqueur interne. Tous les 27 loci microsatellites ont été multiplexés en six panels pour le génotypage comme indiqué dans le Tableau 1. Les génotypes ont ensuite été extraits en utilisant le logiciel GENEMAPPER (V 4. 0).

\section{Analyses statistiques}

Les indices de diversité basiques comme le nombre d'allèles observés par locus (Na), les hétérozygoties observées (Ho) et attendues (He), le déficit en hétérozygotes $F_{I S}$, les $F$-statistiques de Wright, les proportions d'allèles partagés ont été calculés en utilisant le logiciel MICROSATELLITE ANALYZER (MSA) version 3.15 (Dieringer et Schlötterer, 2003). Les écarts des hétérozygoties par rapport à l'équilibre de Hardy-Weinberg (HWE) ont été estimées par des tests exacts d'évaluation d'excès et de déficit hétérozygotes pour chaque marqueur et dans chaque race, tels que mis en œuvre dans GENEPOP (Raymond et Rousset, 1995). L'arbre phylogénique a été construit suivant la méthode du Neighbor Joining en utilisant le logiciel PHYLIP version 3.5 (Felsenstein, 1993). En outre, l'arbre a été visualisé en utilisant MEGA version 6.0 (Tamura et al., 2013). Les probabilités d'assignation ont été calculées à partir des méthodes de 
vraisemblance (Paetkau et al., 1995) et bayésienne (Rannala et Mountain, 1997; Baudoin et Lebrun, 2001) en utilisant le logiciel GENECLASS 2 (Piry et al., 2004). La détection de migrants de première génération a été effectué en utilisant rapport de vraisemblance - $\log$ (L_Home/L_max), via la méthode des fréquences alléliques de Paetkau et al. (1995). Le diagramme de dispersion des estimations de vraisemblance (Baudoin et Lebrun, 2001) pour les individus de chacune des trois races bovines du Niger a été construit en utilisant SPSS version 10.5. Les populations bovines nigériennes ont été testées pour l'équilibre des dérives de mutation selon trois approches statistiques (sign test, standardized differences test et Wilcoxon sign rank test) selon les 3 modèles d'évolution des marqueurs microsatellites utilisant le programme BOTTLENECK (Piry et al., 1999).

Tableau 1: Détails des marqueurs microsatellites.

\begin{tabular}{|c|c|c|c|}
\hline Locus & Numéro Panel & Température d'hybridation & Fluorophores \\
\hline CSSM66 & 1 & $60^{\circ} \mathrm{C}$ & FAM \\
\hline HEL1 & 1 & $56^{\circ} \mathrm{C}$ & HEX \\
\hline INRA63 & 1 & $56^{\circ} \mathrm{C}$ & HEX \\
\hline BM1824 & 2 & $61^{\circ} \mathrm{C}$ & ATTO550 \\
\hline ETH152 & 2 & $60^{\circ} \mathrm{C}$ & FAM \\
\hline HAUT27 & 2 & $54^{\circ} \mathrm{C}$ & HEX \\
\hline INRA05 & 2 & $54^{\circ} \mathrm{C}$ & FAM \\
\hline BM1818 & 3 & $60^{\circ} \mathrm{C}$ & HEX \\
\hline ETH3 & 3 & $63^{\circ} \mathrm{C}$ & FAM \\
\hline HEL9 & 3 & $56^{\circ} \mathrm{C}$ & ATTO550 \\
\hline ILSTS006 & 3 & $54^{\circ} \mathrm{C}$ & FAM \\
\hline TGLA53 & 3 & $55^{\circ} \mathrm{C}$ & HEX \\
\hline HAUT24 & 4 & $53^{\circ} \mathrm{C}$ & HEX \\
\hline HEL5 & 4 & $54^{\circ} \mathrm{C}$ & FAM \\
\hline INRA032 & 4 & $56^{\circ} \mathrm{C}$ & ATTO550 \\
\hline SPS115 & 4 & $61^{\circ} \mathrm{C}$ & FAM \\
\hline ETH185 & 5 & $65^{\circ} \mathrm{C}$ & ATTO550 \\
\hline HEL13 & 5 & $54^{\circ} \mathrm{C}$ & HEX \\
\hline ILSTS05 & 5 & $56^{\circ} \mathrm{C}$ & FAM \\
\hline INRA035 & 5 & $60^{\circ} \mathrm{C}$ & FAM \\
\hline TGLA126 & 5 & $54^{\circ} \mathrm{C}$ & HEX \\
\hline BM2113 & 6 & $63^{\circ} \mathrm{C}$ & FAM \\
\hline ETH10 & 6 & $61^{\circ} \mathrm{C}$ & FAM \\
\hline ETH225 & 6 & $63^{\circ} \mathrm{C}$ & ATTO550 \\
\hline INRA023 & 6 & $58^{\circ} \mathrm{C}$ & ATTO550 \\
\hline TGLA122 & 6 & $58^{\circ} \mathrm{C}$ & HEX \\
\hline
\end{tabular}




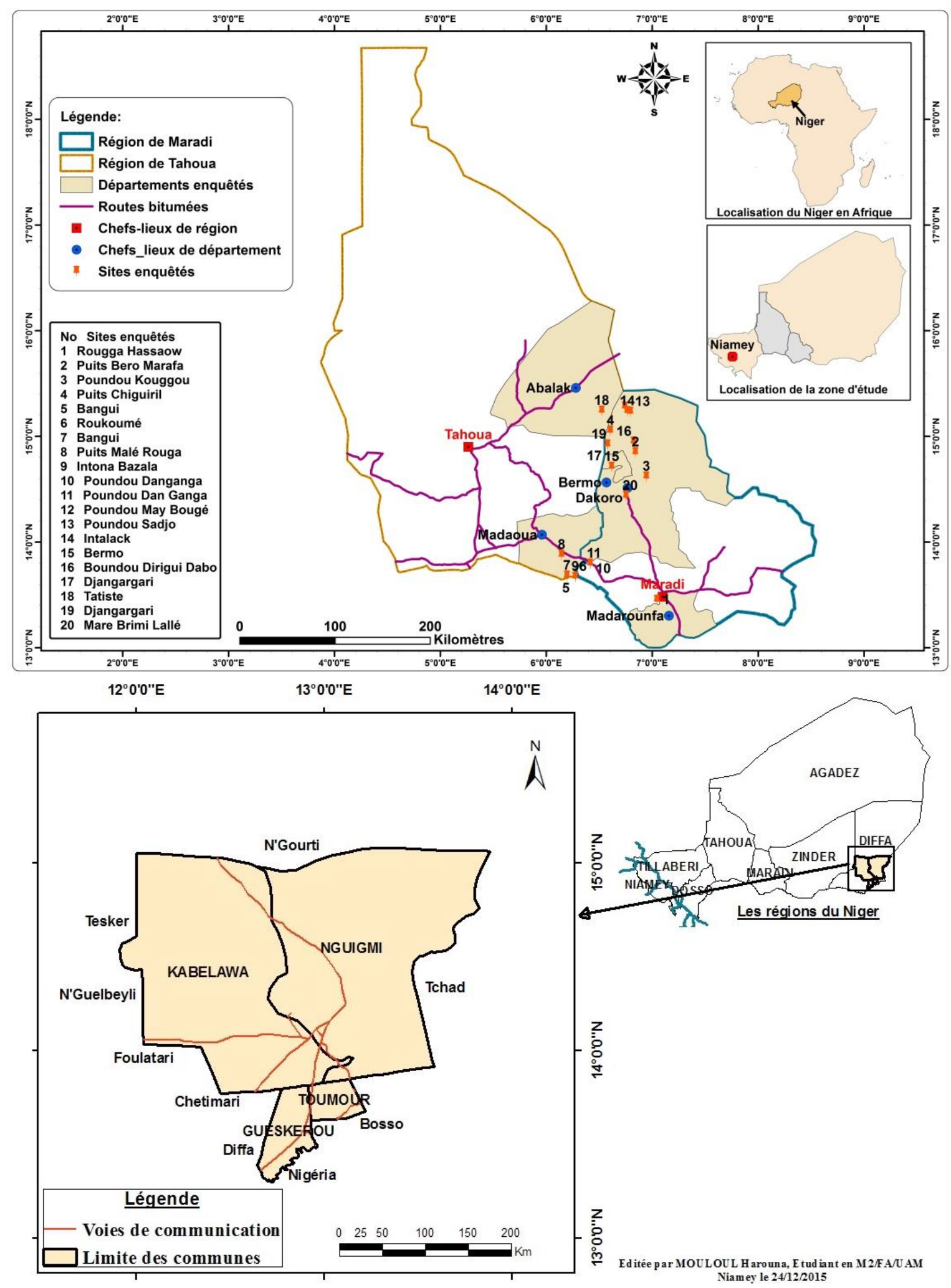

Figure 1 : Sites de l'étude au Niger ((a) Région de Maradi et Tahoua et (b)) Régions de Diffa. 


\section{RESULTATS}

\section{Variabilité génétique et équilibre génétique}

Tous les loci amplifiés ont été polymorphes. Un total de 201 allèles différents a été détecté sur les 27 loci en étude dans les trois (03) populations de zébu Bororo (Tableau 2). Le nombre d'allèles observés par locus varie de 2 (ETH152 et SPS115) à 10 (TGLA53) avec une moyenne de 7,4 allèles observés par locus. D'une localité à l'autre, le nombre moyen d'allèles observés par locus est presque le même. Avec une moyenne de 5,22 allèles, les bororos de la localité de Bangui sont ceux qui présentent le plus faible nombre d'allèles observés par locus contre une moyenne maximale de 5,70 allèles à Bermo. L'indice de richesse allélique (Rt) pour l'ensemble des populations a montré une grande variabilité entre loci passant de 2,704 (SPS115) à 5,04 (CSMM66) pour une moyenne estimée à 3,74 allèles. A l'exception des loci SPS115, ETH3, INRA63 et INRA05, tous les marqueurs ont montré des valeurs de PIC supérieurs à 0,50 pour une moyenne de 0,65 (Tableau 2). Les valeurs d'hétérozygoties observées sont comprises entre 0,25 (ETH3) et 0,94 (BM2113) pour des valeurs moyennes par population supérieures à 0,63 (Tableau 3).

\section{Equilibre et différentiation génétique}

Estimées par population, les valeurs moyennes du $\mathrm{F}_{\mathrm{IS}}$ étaient de 0,$00 ; 0,01$ et $-0,01$ respectivement pour les bororo de Bermo; Bangui et Diffa (Tableau 3). Parmi les 81 combinaisons sous-populations $\mathrm{X}$ locus testées, $4,93 \%$ ont montré une déviation significative $(p<0,05)$ de l'équilibre de HW pour déficit d'hétérozygotes (1 locus pour la sous population de Bermo et 3 loci pour celle de Diffa) et aucune pour excès d'hétérozygotes (Tableau 4). Le $F_{I S}$ moyen pour l'ensemble des populations par locus est de 0,023 . Sur les 27 loci, 12 (soit $44,44 \%$ des loci) ont présenté des valeurs négatives de $\mathrm{F}_{\text {IS }}$ (Tableau 5). Un léger déficit en hétérozygotes $\left(\mathrm{F}_{\mathrm{IT}}=5,7 \%\right)$ a été observé dans la population globale (Tableau 5). Un ensemble de 15 loci ont significativement contribué $(\mathrm{p}<0,05)$ à la différentiation génétique globale entre populations ; le $\mathrm{F}_{\mathrm{ST}}$ global était faible $(0,035)$ et hautement significatif $(\mathrm{p}<0,001)$; (Tableau 5). Les valeurs de $F_{S T}$ par paires de populations sont toutes aussi faibles : 0,04 (entre Bororo de Diffa et de Bangui) ; 0,03 (entre Diffa et Bermo) et 0,02 (entre Bangui et Bermo). Le flux génétique oscille entre 8,54 (entre Bororos de Bangui et de Bermo) et 5,70 (entre Bororos de Bangui et de Diffa); le flux intermédiaire $(7,08)$ étant observé entre bororos de Diffa et de Bermo. Les valeurs du $\mathrm{F}_{\text {ST }}$ par paires de populations sont de 0,04 entre Diffa et Bangui ; 0,03 entre Diffa et Bermo et 0,02 entre Bangui et Bermo (Tableau 6).

\section{Relations phylogénétiques entre populations}

Le dendogramme obtenu montre que les populations se séparent en deux clades (groupe d'animaux descendant d'un ancêtre commun) distincts : un clade formé par les populations de Bangui et de Bermo et un clade formé par la population de Diffa (Figure 2a). Des observations similaires sont faites sur l'arbre phylogénétique avec cependant une disparité de la distribution des individus de Bangui. Les bororo de Bermo et de Diffa formant deux clades bien distincts (Figure 2b).

La représentation des estimations des logarithmes de vraisemblance individuels dans un diagramme de dispersion a révélé des groupes bien distincts avec chevauchement entre individus des populations de Bermo et de Diffa (Figure 3).

\section{Structure génétique \\ Analyse de goulot d'étranglement}

L'analyse du goulot d'étranglement indique un excès en hétérozygotes uniquement sous le modèle IAM dans toutes les populations (Tableau 7). Par contre les populations étudiées n'ont dévié de l'équilibre mutation dérive sous TPM que pour le wilcoxon sign rank test et jamais sous SMM. D'autre part, l'observation des courbes des spectres alléliques (test de l'indicateur modeshift) suit la distribution normale en forme de « $\mathrm{L} »$ (Figure $4 \mathrm{a} ; 4 \mathrm{~b}$ et $4 \mathrm{c}$ ). Ce résultat indique la prédominance des allèles à faible fréquence, ce qui est caractéristique d'une 
population n'ayant pas subi de goulot démographique récent.

Assignation génétique des individus et détection de migrants de première génération

Faisant appel au rapport de vraisemblance - $-\log$ (L_Home/L_max), 10 migrants de première génération ont été détectés dans la population totale via la méthode des fréquences alléliques de Paetkau et al. (1995). Ce sont ainsi 20,83\% des individus de la population globale qui ont été détectés comme migrants de première génération au seuil de 5\% et assignées à des populations où le rapport de vraisemblance était maximal. Le pourcentage moyen d'individus correctement assignés évolue entre $79,1 \%$ et $85,42 \%$ respectivement pour les méthodes de Paetkau et al., (1995) et Baudouin et Lebrun (2001) (Tableau 8). Sur l'ensemble des trois méthodes de classification utilisées, les individus de Bermo et de Diffa sont les mieux classés $(81,25 \%$ pour chacune des deux populations sous Paetkau et al (1995) et sous Baudouin and Lebrun (2001) et $87,5 \%$ (Bermo) ; 81,25\% (Diffa) sous Rannala and Mountain (1997)); les pourcentages d'individus correctement assignés de Bangui étant les plus faibles sur les deux premi.

Tableau 2: Taille des allèles et nombre d'allèles observés par locus et par population.

\begin{tabular}{|c|c|c|c|c|c|c|}
\hline \multirow{2}{*}{ Locus } & \multirow{2}{*}{ Taille allèles } & \multirow{2}{*}{ PIC } & \multirow[t]{2}{*}{$\mathbf{R t}$} & \multicolumn{3}{|c|}{$\begin{array}{c}\text { Nombre d'allèles observé par locus et par } \\
\text { population }\end{array}$} \\
\hline & & & & Bangui & Bermo & Diffa \\
\hline CSRM60 & $87-109$ & 0,598 & 3,588 & 5 & 6 & 7 \\
\hline CSSM66 & $177-197$ & 0,824 & 5,042 & 7 & 8 & 8 \\
\hline HEL1 & 98-106 & 0,622 & 3,414 & 5 & 5 & 3 \\
\hline INRA63 & $175-183$ & 0,465 & 2,746 & 3 & 4 & 4 \\
\hline BM1824 & $183-197$ & 0,687 & 3,566 & 4 & 4 & 4 \\
\hline ETH152 & 189-199 & 0,503 & 2,734 & 2 & 5 & 5 \\
\hline HAUT27 & $140-150$ & 0,691 & 3,789 & 6 & 5 & 5 \\
\hline INRA05 & $134-140$ & 0,496 & 2,878 & 4 & 4 & 4 \\
\hline BM1818 & $256-272$ & 0,708 & 3,928 & 5 & 6 & 7 \\
\hline ETH3 & $99-125$ & 0,442 & 2,832 & 4 & 5 & 6 \\
\hline HEL9 & $155-175$ & 0,828 & 5,019 & 7 & 8 & 8 \\
\hline ILSTS006 & $284-300$ & 0,679 & 4,03 & 7 & 6 & 7 \\
\hline TGLA53 & $153-183$ & 0,754 & 4,468 & 7 & 10 & 8 \\
\hline HAUT24 & $103-125$ & 0,723 & 4,104 & 6 & 7 & 6 \\
\hline HEL5 & $148-178$ & 0,778 & 4,477 & 5 & 5 & 7 \\
\hline INRA032 & $164-208$ & 0,787 & 4,52 & 5 & 7 & 6 \\
\hline SPS115 & $243-255$ & 0,42 & 2,704 & 5 & 6 & 2 \\
\hline ETH185 & $224-252$ & 0,627 & 3,619 & 5 & 5 & 7 \\
\hline HEL13 & $182-192$ & 0,623 & 3,261 & 5 & 4 & 4 \\
\hline ILSTS05 & $178-190$ & 0,694 & 3,827 & 5 & 5 & 5 \\
\hline INRA035 & $99-121$ & 0,659 & 3,658 & 6 & 4 & 6 \\
\hline TGLA126 & $116-128$ & 0,669 & 3,752 & 5 & 5 & 6 \\
\hline
\end{tabular}




\begin{tabular}{lcccccc} 
BM2113 & $118-146$ & 0,81 & 4,822 & 7 & 7 & 8 \\
ETH10 & $207-221$ & 0,709 & 3,916 & 5 & 6 & 6 \\
ETH225 & $140-162$ & 0,655 & 3,602 & 6 & 6 & 3 \\
INRA023 & $199-217$ & 0,603 & 3,352 & 5 & 5 & 5 \\
TGLA122 & $134-160$ & 0,582 & 3,448 & 5 & 6 & 6 \\
\hline MOYENNE & NA & $\mathbf{0 , 6 5}$ & $\mathbf{3 , 7 4}$ & $\mathbf{5 , 2 2}$ & $\mathbf{5 , 7}$ & $\mathbf{5 , 6 7}$ \\
\hline
\end{tabular}

Introduire ici la définition de PIC et Rt, NA=non applicable.

Tableau 3: Hétérozygotie observée (Ho), hétérozygotie attendue (He) et coefficient de consanguinité $\left(\mathrm{F}_{\mathrm{IS}}\right)$ par locus et par population.

\begin{tabular}{|c|c|c|c|c|c|c|c|c|c|}
\hline \multirow[t]{2}{*}{ Locus } & \multicolumn{3}{|c|}{ Bangui } & \multicolumn{3}{|c|}{ Bermo } & \multicolumn{3}{|c|}{ Diffa } \\
\hline & Ho & $\mathrm{He}$ & $\mathrm{F}_{\mathrm{IS}}$ & Ho & $\mathrm{He}$ & $\mathrm{F}_{\mathrm{IS}}$ & Ho & $\mathrm{He}$ & $\mathrm{F}_{\mathrm{IS}}$ \\
\hline CSRM60 & 0,47 & 0,45 & $-0,05$ & 0,63 & 0,65 & 0,02 & 0,75 & 0,73 & $-0,05$ \\
\hline CSSM66 & 0,79 & 0,80 & $-0,01$ & 0,87 & 0,86 & $-0,03$ & 0,88 & 0,85 & $-0,05$ \\
\hline HEL1 & 0,64 & 0,76 & 0,14 & 0,56 & 0,74 & 0,23 & 0,50 & 0,49 & $-0,05$ \\
\hline INRA63 & 0,40 & 0,42 & 0,03 & 0,75 & 0,66 & $-0,16$ & 0,47 & 0,40 & $-0,18$ \\
\hline BM1824 & 0,63 & 0,75 & 0,15 & 0,63 & 0,71 & 0,10 & 0,75 & 0,68 & $-0,12$ \\
\hline ETH152 & 0,69 & 0,51 & $-0,37$ & 0,31 & 0,59 & 0,47 & 0,69 & 0,66 & $-0,07$ \\
\hline HAUT27 & 0,88 & 0,80 & $-0,12$ & 0,60 & 0,74 & 0,18 & 0,63 & 0,67 & 0,05 \\
\hline INRA05 & 0,44 & 0,38 & $-0,19$ & 0,63 & 0,60 & $-0,06$ & 0,50 & 0,61 & 0,17 \\
\hline BM1818 & 0,69 & 0,73 & 0,05 & 0,63 & 0,69 & 0,07 & 0,73 & 0,80 & 0,06 \\
\hline ETH3 & 0,31 & 0,29 & $-0,10$ & 0,25 & 0,24 & $-0,07$ & 0,69 & 0,73 & 0,05 \\
\hline HEL9 & 0,81 & 0,82 & 0,00 & 0,87 & 0,83 & $-0,06$ & 0,93 & 0,87 & $-0,10$ \\
\hline ILSTS006 & 0,56 & 0,72 & 0,21 & 0,88 & 0,73 & $-0,22$ & 0,80 & 0,69 & $-0,18$ \\
\hline TGLA53 & 0,69 & 0,78 & 0,10 & 0,75 & 0,83 & 0,08 & 0,88 & 0,77 & $-0,16$ \\
\hline HAUT24 & 0,56 & 0,75 & 0,24 & 0,75 & 0,81 & 0,06 & 0,56 & 0,74 & 0,23 \\
\hline HEL5 & 0,56 & 0,60 & 0,06 & 0,69 & 0,78 & 0,11 & 0,63 & 0,86 & 0,27 \\
\hline INRA032 & 0,75 & 0,80 & 0,05 & 0,81 & 0,80 & $-0,03$ & 0,88 & 0,81 & $-0,10$ \\
\hline SPS115 & 0,43 & 0,43 & $-0,01$ & 0,56 & 0,56 & $-0,03$ & 0,38 & 0,32 & $-0,22$ \\
\hline ETH185 & 0,64 & 0,64 & $-0,02$ & 0,56 & 0,68 & 0,16 & 0,56 & 0,66 & 0,14 \\
\hline HEL13 & 0,73 & 0,71 & $-0,05$ & 0,67 & 0,70 & 0,03 & 0,56 & 0,63 & 0,09 \\
\hline ILSTS05 & 0,50 & 0,89 & 0,42 & 0,69 & 0,68 & $-0,03$ & 0,75 & 0,77 & 0,01 \\
\hline INRA035 & 0,71 & 0,68 & $-0,08$ & 0,56 & 0,60 & 0,04 & 0,63 & 0,71 & 0,11 \\
\hline TGLA126 & 0,69 & 0,73 & 0,03 & 0,56 & 0,55 & $-0,04$ & 0,81 & 0,79 & $-0,04$ \\
\hline BM2113 & 0,88 & 0,81 & $-0,09$ & 0,88 & 0,82 & $-0,09$ & 0,94 & 0,84 & $-0,13$ \\
\hline ETH10 & 0,81 & 0,69 & $-0,19$ & 0,81 & 0,78 & $-0,06$ & 0,80 & 0,79 & $-0,03$ \\
\hline ETH225 & 0,69 & 0,68 & $-0,03$ & 0,75 & 0,77 & 0,01 & 0,69 & 0,65 & $-0,08$ \\
\hline INRA023 & 0,71 & 0,65 & $-0,13$ & 0,60 & 0,48 & $-0,27$ & 0,73 & 0,76 & 0,02 \\
\hline TGLA122 & 0,44 & 0,43 & $-0,04$ & 0,63 & 0,62 & $-0,02$ & 0,69 & 0,72 & 0,03 \\
\hline Moyenne & 0,63 & 0,66 & $\mathbf{0 , 0 0}$ & 0,66 & 0,68 & $\mathbf{0 , 0 1}$ & $\mathbf{0 , 7 0}$ & $\mathbf{0 , 7 0}$ & $-0,01$ \\
\hline
\end{tabular}


Tableau 4: Excès et déficit d'hétérozygotie sous équilibre d'Hardy-Weinberg.

\begin{tabular}{lcccccc}
\hline \multirow{2}{*}{ Locus } & \multicolumn{2}{c}{ Déficit d'hétérozygotes } & \multicolumn{3}{c}{ Excès d'hétérozygotes } \\
\cline { 2 - 7 } & Bangui & Bermo & Diffa & Bangui & Bermo & Diffa \\
\hline CSRM60 & 0,7183 & 0,4667 & 0,3171 & 0,6502 & 0,6578 & 0,7001 \\
CSSM66 & 0,3977 & 0,4377 & 0,7562 & 0,6458 & 0,5833 & 0,3613 \\
HEL1 & 0,1057 & 0,073 & 0,6129 & 0,9285 & 0,9358 & 0,5357 \\
INRA63 & 0,5954 & 0,7261 & 1 & 0,774 & 0,3185 & 0,4046 \\
BM1824 & 0,23 & 0,1718 & 0,6695 & 0,7934 & 0,847 & 0,3598 \\
ETH152 & 0,9747 & 0,0117 & 0,6791 & 0,1834 & 0,9953 & 0,4681 \\
HAUT27 & 0,8449 & 0,1854 & 0,0436 & 0,2283 & 0,8462 & 0,9576 \\
INRA05 & 1 & 0,6193 & 0,2654 & 0,4351 & 0,5124 & 0,8234 \\
BM1818 & 0,3838 & 0,405 & 0,2977 & 0,735 & 0,6983 & 0,755 \\
ETH3 & 1 & 1 & 0,4061 & 0,6941 & 0,8092 & 0,6355 \\
HEL9 & 0,5634 & 0,7434 & 0,6581 & 0,5447 & 0,415 & 0,3628 \\
ILSTS006 & 0,2257 & 0,9848 & 0,9665 & 0,7892 & 0,0905 & 0,1841 \\
TGLA53 & 0,3493 & 0,302 & 0,929 & 0,6986 & 0,7528 & 0,1333 \\
HAUT24 & 0,1793 & 0,4186 & 0,0884 & 0,8263 & 0,6585 & 0,9471 \\
HEL5 & 0,4371 & 0,24 & 0,0063 & 0,7168 & 0,7925 & 0,994 \\
INRA032 & 0,3385 & 0,6329 & 0,6852 & 0,725 & 0,4722 & 0,3438 \\
SPS115 & 0,6298 & 0,6692 & 1 & 0,7641 & 0,6002 & 0,6261 \\
ETH185 & 0,6147 & 0,3663 & 0,0262 & 0,569 & 0,6939 & 0,9808 \\
HEL13 & 0,4453 & 0,5531 & 0,3919 & 0,5644 & 0,5314 & 0,7497 \\
ILSTS05 & 0,0674 & 0,5534 & 0,4809 & 0,9904 & 0,5436 & 0,5289 \\
INRA035 & 0,7059 & 0,4444 & 0,5515 & 0,4701 & 0,7089 & 0,4719 \\
TGLA126 & 0,4924 & 0,6931 & 0,477 & 0,6264 & 0,5282 & 0,5532 \\
BM2113 & 0,7584 & 0,6284 & 0,9417 & 0,3264 & 0,4119 & 0,2059 \\
ETH10 & 0,9139 & 0,7156 & 0,6607 & 0,1793 & 0,3542 & 0,4247 \\
ETH225 & 0,6505 & 0,1703 & 0,6171 & 0,5603 & 0,8498 & 0,5177 \\
INRA023 & 0,7316 & 1 & 0,4202 & 0,3877 & 0,1795 & 0,6482 \\
TGLA122 & 0,6883 & 0,6619 & 0,5341 & 0,6768 & 0,5136 & 0,5285 \\
\hline & & & & & & \\
\hline
\end{tabular}

Tableau 5: F-statistiques par locus*.

\begin{tabular}{lrrrr}
\hline LocuS & $\mathbf{F}_{\text {ST }}$ & $\mathbf{F}_{\text {IT }}$ & $\mathbf{F}_{\text {IS }}$ & P-values \\
\hline CSRM60 & 0,04 & 0,032 & $-0,0084$ & 0,026 \\
CSSM66 & 0,025 & 0,016 & $-0,009$ & 0,034 \\
HEL1 & 0,04 & 0,17 & 0,14 & 0,035 \\
INRA63 & 0,04 & $-0,05$ & $-0,1$ & 0,056 \\
BM1824 & 0,06 & 0,12 & 0,07 & 0,01 \\
ETH152 & $-0,002$ & 0,04 & 0,04 & 0,41 \\
HAUT27 & 0,006 & 0,054 & 0,05 & 0,25 \\
INRA05 & 0,037 & 0,051 & 0,014 & 0,06 \\
BM1818 & 0,033 & 0,11 & 0,08 & 0,04 \\
ETH3 & 0,145 & 0,152 & 0,008 & 0,0001 \\
HEL9 & 0,027 & $-0,008$ & $-0,04$ & 0,024 \\
ILSTS006 & $-0,006$ & $-0,05$ & $-0,04$ & 0,53 \\
TGLA53 & $-0,005$ & 0,021 & 0,026 & 0,54 \\
HAUT24 & $-0,0054$ & 0,183 & 0,188 & 0,55 \\
HEL5 & 0,106 & 0,26 & 0,171 & 0,0001 \\
INRA032 & 0,034 & 0,021 & $-0,012$ & 0,024 \\
SPS115 & 0,006 & $-0,04$ & $-0,043$ & 0,207
\end{tabular}




\begin{tabular}{lrrrr} 
ETH185 & 0,013 & 0,14 & 0,13 & 0,14 \\
HEL13 & 0,025 & 0,06 & 0,036 & 0,103 \\
ILSTS05 & 0,009 & 0,071 & 0,063 & 0,12 \\
INRA035 & 0,095 & 0,14 & 0,05 & 0,0007 \\
TGLA126 & 0,053 & 0,052 & $-0,001$ & 0,011 \\
BM2113 & 0,027 & $-0,06$ & $-0,09$ & 0,04 \\
ETH10 & 0,009 & $-0,06$ & $-0,074$ & 0,22 \\
ETH225 & 0,028 & 0,013 & $-0,015$ & 0,076 \\
INRA023 & 0,076 & $-0,003$ & $-0,09$ & 0,005 \\
TGLA122 & 0,057 & 0,07 & 0,014 & 0,009 \\
\hline TOTAL & $\mathbf{0 , 0 3 5}$ & $\mathbf{0 , 0 5 7}$ & $\mathbf{0 , 0 2 3}$ & $\mathbf{0 , 0 0 0 1}$ \\
\hline
\end{tabular}

Tableau 6: Valeurs de $\mathrm{F}_{\mathrm{ST}}$ en dessous de la diagonale et celle du flux de gènes $(\mathrm{Nm})$ au-dessus de la diagonale.

\begin{tabular}{lccc}
\hline & Bororo Diffa & Bororo Bangui & Bororo Bermo \\
\hline Bororo Diffa & - & 5,705358632 & 7,08869547 \\
Bororo Bangui & $0,041979^{* * *}$ & - & 8,54507476 \\
Bororo Bermo & $0,034066^{* * *}$ & $0,028425^{* * *}$ & - \\
\hline
\end{tabular}

Tableau 7 : Tests quantitatifs de réduction de la taille effective des sous-populations du bovin zébu bororo de Bangui, Bermo et Diffa au Niger.

\begin{tabular}{lllllllllll} 
& & \multicolumn{3}{c}{ Bangui } & \multicolumn{3}{c}{ Bermo } & \multicolumn{3}{c}{ Diffa } \\
\cline { 2 - 9 } Tests & & IAM & TPM & SMM & IAM & TPM & SMM & IAM & TPM & SMM \\
\hline Sign test & $\begin{array}{l}\text { Nombre attendu de loci } \\
\text { avec excès } \\
\text { d'hétérozygotie }\end{array}$ & 15,9 & 16,16 & 15,68 & 15,99 & 16 & 15,98 & 15,76 & 15,97 & 16,05 \\
& $\begin{array}{l}\text { Nombre observéy de } \\
\text { loci avec excès } \\
\text { d'hétérozygotie }\end{array}$ & 18 & 15 & 10 & 19 & 18 & 11 & 22 & 19 & 12 \\
& P-value & 0,26 & 0,39 & 0,021 & 0,16 & 0,28 & 0,04 & 0,009 & 0,16 & 0,08 \\
\hline Standardized & T-value & 1,54 & $-0,86$ & $-4,11$ & 1,61 & $-0,75$ & $-4,53$ & 3,08 & 1,08 & $-1,76$ \\
\hline $\begin{array}{l}\text { differences } \\
\text { test }\end{array}$ & P-value & 0,061 & 0,2 & 0 & 0,053 & 0,22 & 0 & 0,001 & 0,14 & 0,04 \\
\hline $\begin{array}{l}\text { Wilcoxon } \\
\text { sign rank } \\
\text { test }\end{array}$ & $\begin{array}{l}\text { P-value (one tail for } \\
\text { He excess) }\end{array}$ & 0,085 & 0,57 & 0,99 & 0,01 & 0,48 & 0,97 & 0,0001 & 0,045 & 0,81 \\
\hline
\end{tabular}


Tableau 8: Test d'assignation des génotypes des sous-populations du bovin zébu bororo de Bangui, Bermo et Diffa au Niger.

\begin{tabular}{|c|c|c|c|c|c|c|c|}
\hline \multirow[b]{2}{*}{ Races } & \multirow[b]{2}{*}{$\mathbf{N}$} & \multicolumn{2}{|c|}{ Paetkau et al. (1995) } & \multicolumn{2}{|c|}{ Rannala and Mountain (1997) } & \multicolumn{2}{|c|}{ Baudouin and Lebrun (2001) } \\
\hline & & $\begin{array}{l}\text { Nombre } \\
\text { d'assignation } \\
\text { correcte }\end{array}$ & $\begin{array}{l}\% \\
\text { d'assignation } \\
\text { correcte }\end{array}$ & $\begin{array}{l}\text { Nombre } \\
\text { d'assignation } \\
\text { correcte }\end{array}$ & $\begin{array}{l}\% \text { d'assignation } \\
\text { correcte }\end{array}$ & $\begin{array}{l}\text { Nombre } \\
\text { d'assignation } \\
\text { correcte }\end{array}$ & $\begin{array}{l}\% \text { d'assignation } \\
\text { correcte }\end{array}$ \\
\hline Bangui & 16 & 12 & 75 & 12 & 75 & 15 & 93,75 \\
\hline Bermo & 16 & 13 & 81,25 & 14 & 87,5 & 13 & 81,25 \\
\hline Diffa & 16 & 13 & 81,25 & 13 & 81,25 & 13 & 81,25 \\
\hline Total & 48 & 38 & 79,16 & 39 & 81,25 & 41 & 85,42 \\
\hline
\end{tabular}

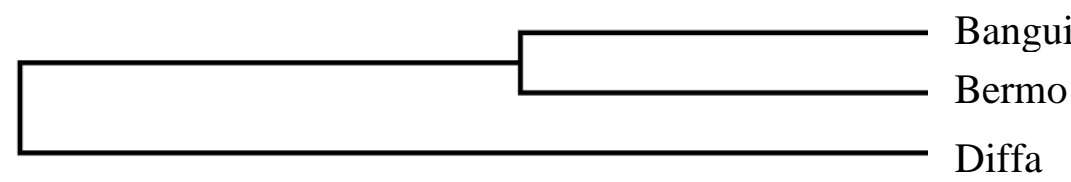

(a)

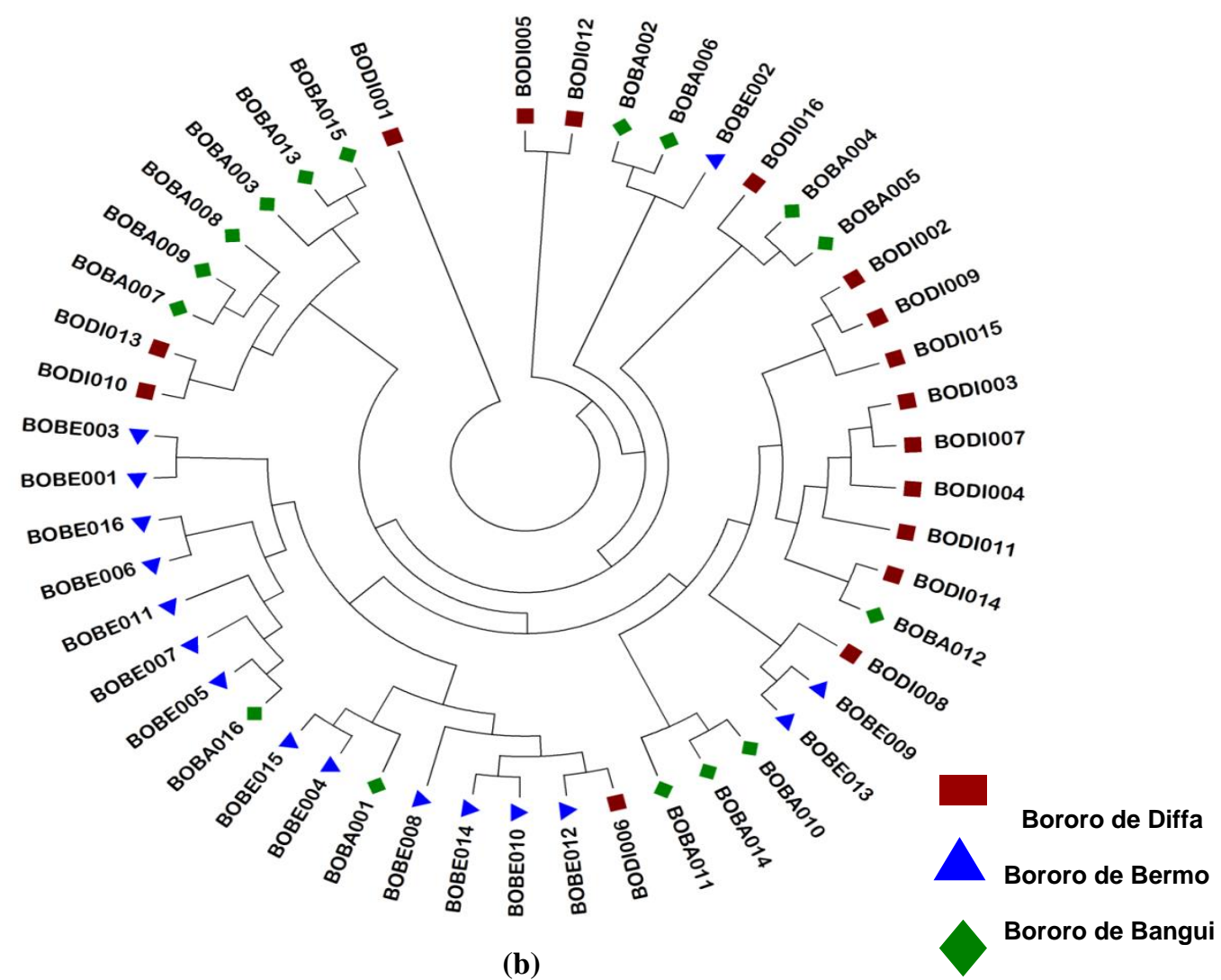

Figure 2 : Arbre phylogénique de Neighbor Joining sur la base des distances entre sous-populations (a) et inter-individuelles (b) dans la race bovin zébu bororo de Bangui, Bermo et Diffa au Niger. 


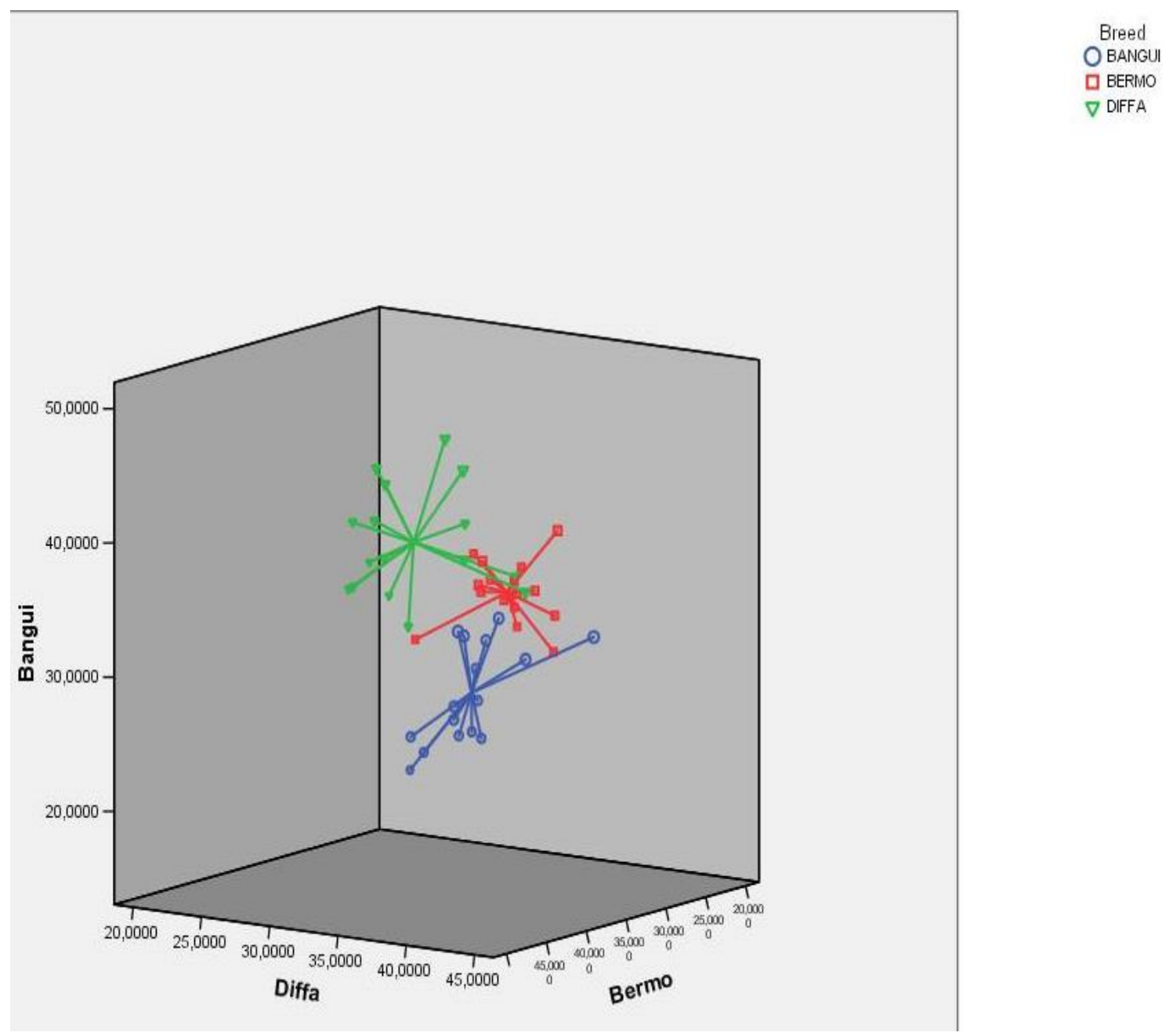

Figure 3: Digramme de dispersion (Baoudouin and Lebrun, 2001) pour les différents individus des sous-populations du bovin zébu bororo de Bangui, Bermo et Diffa au Niger.

(a) Bangui

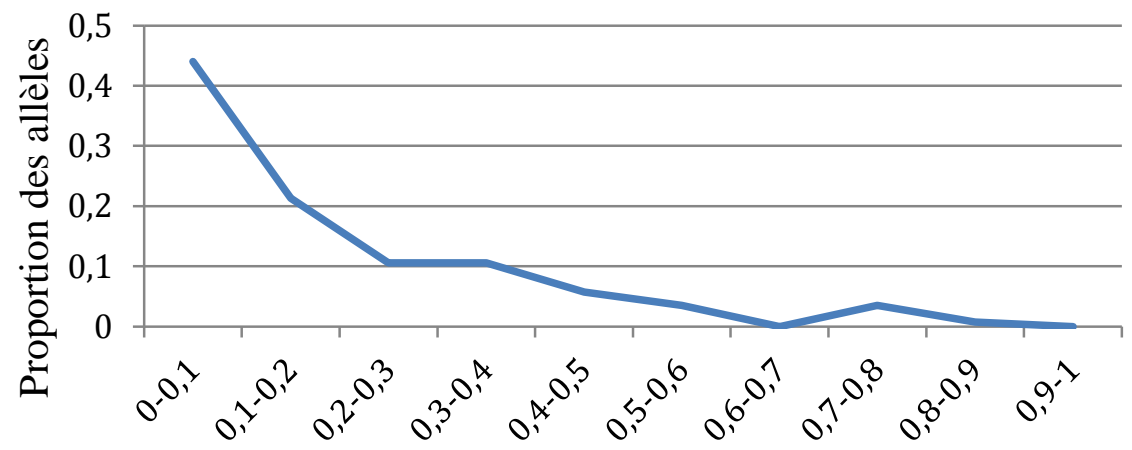

Classe des fréquences alléliques 
(b) Bermo

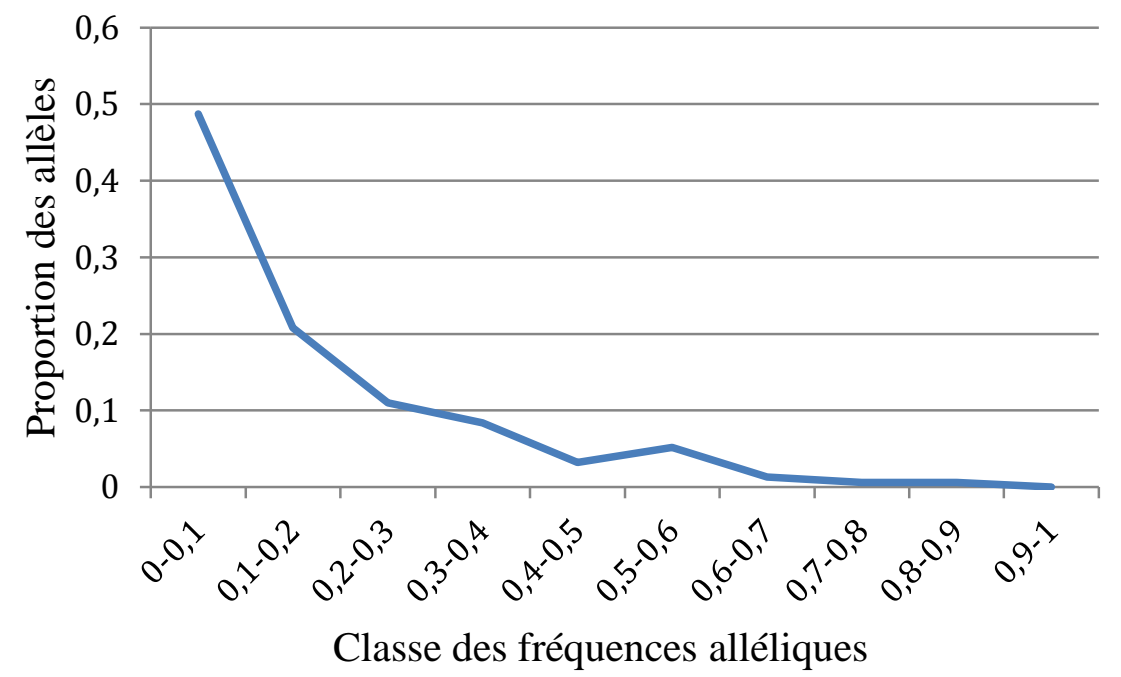

(c) Diffa

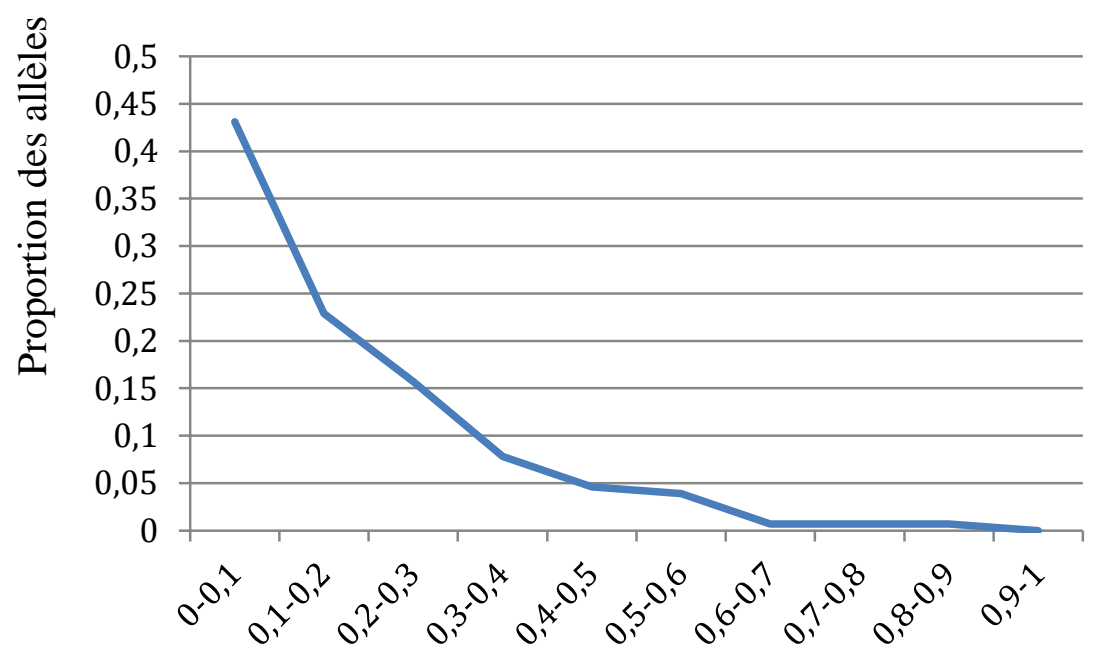

Classe des fréquences alléliques

Figure 4: Test qualitatif de réduction de la taille effective des populations (L-shaped distribution des fréquences alléliques de la courbe normale L) dans les (a) sous-populations de Bangui (b) souspopulations de Bermo et (c) sous-populations de Diffa. 


\section{DISCUSSION}

\section{Variabilité et équilibre génétique}

Un panel de 27 marqueurs microsatellites choisis parmi ceux recommandés par la FAO a été utilisé pour l'étude de la variabilité génétique de zébus bororo provenant de trois localités différentes. Avec une valeur moyenne du contenu informatif du polymorphisme (PIC) de 0,65 , les marqueurs sélectionnés ont montré une capacité informative (PIC) très importante. Des moyennes de PIC plus élevés ont été rapportés dans des études de caractérisation bovines au Sénégal $(0,75)$ (Ndiaye et al., 2015) avec l'utilisation de 11 marqueurs microsatellites sur quatre races bovines du pays (Zébu Gobra, Zébu Maure, Zébu Djakoré et taurin N'dama) et au Cameroun (Ngono Ema et al., 2014) également sur quatre races avec 13 loci microsatellites dans les même conditions d'amplification génique. A l'exception des loci (SPS115, ETH3, INRA63 et INRA05) toutes les valeurs du PIC étaient supérieures à 0,5 , traduisant là encore une capacité informative élevée des marqueurs choisis. La valeur la plus basse était de 0,42 (SPS115). Toutefois, il a été démontré que des valeurs de PIC comprises entre 0,25 et moins de 0,5 donnent des informations fiables et exploitables dans des études de caractérisation (Vaiman et al., 1994 ; Ya-Bo et al., 2006). Le nombre moyens d'allèles observés par locus $(5,22)$ est comparable à celui rapportée dans les populations de taurins Ndama au Mali $(\mathrm{Na}=5,63)$ (Kaboré, 2012). Par ailleurs, ces estimations restent inférieures à celles de races bovines de Centrafrique $(\mathrm{Na}=10)$ (Moazami-Goudarzi et al., 2001), du Sénégal $(10,45)$ (Ndiaye et al., 2015) et du Cameroun (Ngono Ema et al., 2014). Une moyenne de 3,74 allèles est présente par locus. Ce niveau de richesse allélique avoisine le standard de la FAO (2004) qui exige une moyenne d'au moins 4 allèles distincts et dénote d'une variabilité génétique moyenne. Cet écart des standards peut s'expliquer par le faible nombre d'individus échantillonnés (48), la richesse allélique étant dépendante de la taille de la population. Les moyennes d'hétérozygoties observées (Ho) par population ont variées entre 0,63 (Bangui) et 0,70 (Diffa). Ces résultats traduisent une diversité génique élevée dans ces 3 populations. L'ensemble de ces résultats montre qu'il y a une forte variabilité génétique chez le zébu bororo du Niger. Certains auteurs tels que Thevenon et al., (2007) expliquent cette diversité génétique importante par l'existence d'une grande taille efficace de populations consécutives aux effets d'un élevage de type extensif marqué par l'absence de sélection et dominée par des pratiques pastorales telles que le nomadisme ou la transhumance.

\section{Equilibre et différentiation génétique}

En dépit du déficit en hétérozygotes observés dans la majorité des loci, aucune déviation de l'équilibre de Hardy-Weinberg n'a été observée ni dans les sous populations ni dans la population globale. Cette observation est confortée par les valeurs moyennes du $\mathrm{F}_{\mathrm{IS}}$ qui mettent en exergue un équilibre panmictique quasi parfait $\left(\mathrm{F}_{\mathrm{IS}}=0\right)$.

Un déficit d'hétérozygotie globale $\left(\mathrm{F}_{\mathrm{IT}}\right)$ de $5,7 \%$ a été observé dans la population totale. Toutefois ce déficit reste inférieure aux $11,7 \%$ et $14,6 \%$ décrits respectivement dans les races de zébu et de taurin au Sénégal (Ndiaye et al., 2015) et en Mozambique (Bessa et al., 2009). Quant au $\mathrm{F}_{\text {ST }}$ global, il est de 0,035 et est proche de 0,05 . De tels niveaux de $\mathrm{F}_{\mathrm{ST}}$ sont considérés par les investigateurs comme faibles et interprétés comme traduisant une faible différenciation génétique des populations étudiés (Wright, 1978; Balloux et Lugon-Moulin, 2002). Se basant en effet sur les valeurs $\mathrm{du} \mathrm{F}_{\mathrm{ST}}$, seulement $3,5 \%$ de la variabilité totale serait due aux différences entre populations. Ces résultats démontrent bien l'appartenance de ces trois sous populations à la même race. Des niveaux similaires de différenciation génétique ont été rapportés dans les populations de bororo au Nigéria et au Cameroun (Ibeagha-Awemu et Erhardt, 2006). Toutefois, ces niveaux de différenciation génétique restent inférieurs à ceux retrouvés dans la littérature chez les bovins au Cameroun $(6,1 \%)$ (Ngono Ema et al., 2014) , au Soudan (7\%) (Hussein et al., 
2015), en Asie (11,7) (Shi et al., 2010) et en Europe (11,2) (MacHugh et al., 1997).

Les valeurs de $F_{\text {ST }}$ par paires de populations montrent que la différenciation génétique entre les trois populations est très faible. Les valeurs de $\mathrm{F}_{\mathrm{ST}}$ mettent en évidence l'existence de flux de gènes importants entre les trois populations qui tendent à s’homogénéiser. Les valeurs du flux génique $(\mathrm{Nm})$ entre paires de populations ont en effet révélés l'existence d'importants échanges alléliques entre les trois populations. Le flux génique est d'autant plus important que les populations sont faiblement différenciées (8,54: Bangui-Bermo ; 5,70 : Bangui-Diffa); 7,08 : Diffa-Bermo). Ces observations mettent en évidence un système d'élevage marqué par une reproduction panmictique. Les études précédentes que nous avons conduites sur la gestion des bororo du Niger renforcent cette hypothèse et font état d'un l'élevage pratiqué essentiellement selon un mode transhumant. En outre, la quasi-totalité des effectifs de bororo au Niger sont détenues par les «Wodaabes» peulhs transhumants qui ne sont retrouvés que dans la zone d'étude. Ces éleveurs partagent les mêmes zones de pâturage et les mouvements des animaux sont dictés par les saisons (notamment pluvieuses) qui conditionnent la disponibilité herbagère sur les zones de pâture. Cette observation est commune aux races africaines qui présentent de faibles valeurs de $F_{S T}$ et sont pour l'essentiel élevés suivant un système d'élevage transhumant (Dayo et al., 2009; Thevenon et al., 2007).

\section{Relations phylogénétiques entre populations}

Le dendogramme obtenu montre clairement la proximité génétique entre bororo de Bangui et Bermo qui forment un clade à part. Ce résultat confirme l'existence de flux génique important entre ces deux populations qui tendent à être assimilées à une seule population. En revanche, bien que des échanges alléliques existent entre les populations de Diffa et les autres, elle forme un clade à part révélant du faible degré d'introgression de cette population par les autres. Des observations similaires sont retrouvées sur le scatter plot et sur l'arbre phylogénétique.

\section{Structure génétique}

L'analyse de goulot d'étranglement génétique et l'assignation des populations a permis d'inférer leurs structures génétiques. L'analyse du goulot d'étranglement démographique indique un excès en hétérozygotes uniquement sous le modèle IAM dans toutes les populations. Face à des résultats similaires, certains auteurs émettent une réserve quant à la convenance pour les microsatellites du modèle de mutation (IAM) qui a été recommandé par Luikart et al., (1998) pour des analyses sur des allozymes. Toutefois, les populations étudiées ont rarement dévié de l'équilibre mutation dérive sous TPM et jamais sous SMM. Il est démontré que si un goulot d'étranglement a réellement lieu dans une population, on le détectera très fortement avec l'hypothèse IAM, moyennement avec le TPM et faiblement avec le SMM (De Meeûs et al., 2010). Au regard de nos différents résultats, nous pouvons donc conclure qu'aucune des trois populations ici en étude n'a subi de goulot d'étranglement récent. La confirmation est donnée avec le test Mode Shift qui est un descripteur qualitatif de la distribution des fréquences alléliques (mode-shift indicateur en anglais) permettant de discriminer les populations en goulot des populations stables. Dans le spectre des fréquences alléliques visualisé par cette méthode, les allèles microsatellites sont organisés en 10 classes alléliques permettant de vérifier si la distribution des fréquences allélique suit la forme « $\mathrm{L}$ » et que les allèles à faible fréquence $(0,01-0,1)$, sont les plus abondants ; ce qui est caractéristique des populations évoluant sous équilibre mutation dérive et n'ayant donc pas subit de goulot d'étranglement. Les courbes de spectres alléliques des différentes races présentent toutes une forme en " $\mathrm{L}$ ", caractéristique indiquant une évolution sous équilibre mutation-dérive de chacune de nos trois populations. Ces résultats sont similaires à 
ceux rapportés dans les races bovines du Sénégal (Ndiaye et al., 2015).

En ce qui concerne l'assignation des populations, au moins $75 \%$ des individus sont correctement assignés dans leurs populations d'origine en utilisant les méthodes de classification basées sur les fréquences alléliques et celles exploitant l'approche Bayésienne. Ces résultats mettent en évidence la performance de ces méthodes comparativement à d'autres basées sur les distances (Cornuet et al., 1999). La détection de migrants de première génération à travers la méthode de vraisemblance met en évidence l'existence de flux de gènes importants et datant de plusieurs générations entre les trois (3) populations.

\section{Conclusion}

L'objectif de cette étude est d'évaluer la diversité et d'inférer la structure génétique de la race bovine bororo du Niger. L'utilisation du polymorphisme de 27 marqueurs microsatellites a permis d'une part de déterminer les indices de diversité génétique au sein de trois populations bovines de race bororo au Niger. Les indices de diversité mettent en évidence l'existence d'une importante diversité génétique. D'autre part, sur le plan structurel, ces trois populations sont faiblement différenciées génétiquement en raison de l'existence de flux génique important entre ces trois populations. Si pour le moment ces échanges n'influent pas sur la structure de ces trois populations, des mesures sont toutefois à mettre en œuvre pour préserve la diversité génétique du bovin bororo de ces trois localités. La constitution de noyau de souches pures de chacun des trois écotypes peut constituer un bon moyen à court et à long terme pour préserver la diversité génétique des bororo dans ces trois localités du Niger.

\section{CONFLIT D'INTERETS}

Les auteurs déclarent qu'il n'existe aucun conflit d'intérêts lié à la présente étude.

\section{CONTRIBUTIONS DES AUTEURS}

AT, MI, HHT et KP ont conçu le projet; MMAM, MG, ASRT, MH, MA, AS, MS et YA ont collecté les données; MMAM, MG, MH, ASRT, MS et AS ont effectué l'extraction de l'ADN; MS et RP ont réalisé la PCR et le génotypage; KP, AT, ASRT, MMAM, MI et AS ont analysé les données et rédigé le manuscrit.

\section{REMERCIEMENTS}

Cette étude a été financée par le Programme de productivité agricole en Afrique de l'Ouest (PPAAO/WAAPP) -Niger et le projet de coopération technique (BKF5017) sur «L'utilisation de méthodes modernes de sélection animale, d'outils nucléaires et génomiques pour améliorer la production laitière dans les systèmes de production à faibles niveaux d'intrants», financés par l'Agence internationale de l'énergie atomique (AIEA).

\section{REFERENCES}

Alhassane A, Soumana I, Chaibou I, Karim S, Mahamane A, Saadou M. 2016. Productivité, valeur pastorale et capacité de charge des parcours naturels de la région de Maradi, Niger. Int. J. Biol. Chem. Sci., 12(4): 1705-1716. DOI: https://dx.doi.org/10.4314/ijbcs.v12i4.15

Balloux F, Lougon-Moulin N. 2002. The estimation of population differenciation with microsatellite markers. Mol. Ecol., 11: $155-165$. DOI: $10.1046 / j .0962-$ 1083.2001.01436.x

Bessa I, Pinhero I, Matola M, Dzama K, Rocha A, Alexendrino P. 2009. Genetic diversity and relationships among indigenous Mozambican cattle breeds. $S$. Afr. J. Anim. Sci., 39: 61-67. DOI: http://dx.doi.org/10.4314/sajas.v39i1.435 $48 \% 20$

Boma S, Nuto Y, Dayo GK, Bonfoh B, N'Feide T. 2018. Caractérisation morpho-biométrique des populations bovines locales sans bosse du Togo. Int. J. Biol. Chem. Sci., 12(1): 431-445. DOI: https://dx.doi.org/10.4314/ijbcs.v12i1.34

Clarck AG. 1998. Mutation-selection balance with multiples alleles. Genetica, 102: 41- 
47. DOI: https://doi.org/10.1046/j.09621083.2001.01436.x

Cornuet JM, Piry S, Luikart G, Estoup A, Solignac M. 1999. New methods employing multilocus genotypes to select or exclude populations as origins of individuals. Genetics, 153(4): 19892000. PMID: 10581301

Dayo GK, Thevenon S, Berthier D, MoazamiGoudrazi K, Denis C, Cuny G. 2009. Detection of selection signatures within candidate regions underlying trypanotolerance in outbred cattle populations. Mol. Ecol., 18 : 1801-1813. DOI: 10.3389/fgene.2015.00137

De Meeûs T, Koffi BB, Barré N, De GarineWichatitsky M, Chevillon C. 2010. Swift sympatric adaptation of a species of cattle tick to a new deer host in New Caledonia. Infection, Genetics and Evolution, 10: 976-983. DOI: 10.1016/j.meegid.2010.06.005

Dieringer D, Schlotterer C. 2003. MICROSATELLITE ANALYZER (MSA): a platform independent analysis tool for large microsatellite data sets. Mol. Ecol. Notes, 3: 167-169. DOI: 10.1046/j.1471-8286.2003.00351.x

Douffissa A. 1993. L'élevage bovin dans le Mbéré (Adamaoua Camerounais). Editions ORSTOM, Collection Etudes et Thèses, Paris.

Felsenstein J. 1993. PHYLIP: Phylogeny inference package, version 3.5. Department of Genetics, Washington University, Seattle, Washington.

Food and Agricultural Organisation of the United Nations. 2004. Secondary Guidelines for development of National Farm Animal Genetic Resources using reference microsatellites. http://dad.fao.org./en/refer/library/guideli ne/marker.pdf.

Habou AZ, Boubacar KM, Adam T. 2016. Les systèmes de productions agricoles du Niger face au changement climatique : défis et perspectives. Int. J. Biol. Chem. Sci., 10(3): 1262-1272. DOI: 10.4314/ijbcs.v10i3.28
Hussein IH, Alam SS, Makkawi AAA, SidAhmed SEA, Abdoon AS, Hassanane MS. 2015. Genetic Diversity Between and Within Sudanese Zebu Cattle Breeds Using Microsatellite Markers. Research in Genetics, 2015: 1-16. DOI: 10.5171/2015.135483

Ibeagha-Awemu EM, Erhardt G. 2006. An evaluation of genetic diversity indices of the Red Bororo and White Fulani cattle breeds with different molecular markers and their implications for current and future improvement options. Trop. Anim. Health Prod., 38(5): 431-441. DOI : https://doi.org/10.1007/s11250-0064347-y

Institut National de la statistique (INS), 2008. Annuaire statistique 2008-2012. Ministère des Finances, Niger. 238p.

Kaboré M. 2012. Etude de la diversité génétique des taurins baoulés du Burkina Faso à l'aide de marqueurs microsatellites. Mémoire, Université de OUAGADOUGOU, $68 \mathrm{p}$.

Kassa KS, Ahounou S, Dayo G-K, Salifou C, Issifou MT, Dotché I, Gandonou PS, Yapi-Gnaore V, Koutinhouin B, Mensah GA, Youssao IAK. 2016. Performances de production laitière des races bovines de l'Afrique de l'Ouest. Int. J. Biol. Chem. Sci., 10(5): 2316-2330. http://dx.doi.org/10.4314/ijbcs.v10i5.29

Luikart G, Allendorf F, Cornuet J, Sherwin W. 1998. Distortion of allele frequency distributions provides a test for recent population bottlenecks. J Hered, 89: 238-247. DOI: 10.1093/jhered/89.3.238

MacHugh DE, Shriver MD, Loftus RT, Cunningham P, Bradley DG. 1997. Microsatellites DNA Variation and the Evolution, Domestication and Phylogeography of Taurine and Zebu cattle (Bos Taurus and Bos indicus). Genetics, 146: 1071-1086.

Moazami-Goudarzi K, Belemsaga DMA, Geriotti G, Laloë D, Fagbohoun NT, Kouagou I, Sidibe I, Codjia V, Crimella MC, Grosclaude F, Toure SM. 2001. Caractérisation de la race bovine Somba à l'aide de marqueurs moléculaires. Elev. 
Med. Vét. Pays Trop., 54(2) : 129-138. DOI: 10.19182/remvt.9791

Ndiaye NP, Sow A, Dayo GK, Ndiaye S, Sawadogo GJ, Sembène M. 2015. Genetic diversity and phylogenetic relationships in local cattle breeds of Senegal based on autosomal microsatellite markers. Veterinary World, 8: 994-1005. DOI: 10.14202/vetworld.2015.994-1005

Ngono Ema PJ, Manjeli Y, Meutchieyié F, Keambou C, Wanjala B, Desta AF, Ommeh S, Skilton R, Djikeng A. 2014. Genetic diversity of four Cameroonian indigenous cattle using microsatellite markers. Journal of Livestock Science, 5: 9-17.

Paetkau D, Calvert W, Sterling I, Strobeck C. 1995. Microsatellite analysis of population structure in Canadian polar bears. Mol. Ecol., 4: 347-354. DOI: 10.1111/j.1365-294X.1995.tb00227.x

Piry S, Alapetite A, Cornuet JM, Paetkau D, Baudouin L, Estoup A. 2004. A Software for Genetic Assignment and First-Generation Migrant Detection. $J$. Hered., 95: 536-539. DOI: 10.1093/jhered/esh074

Rannala B, Mountain JL. 1997. Detecting immigration by using multilocus genotypes. Proc. Natl. Acad. Sci. USA, 94:

9197-

920. DOI:10.1073/pnas.94.17.9197

Raymond M, Rousset F. 1995. GENEPOP (version 1.2): population genetics software for exact tests and ecumenicism. J. Hered., 86: 248-249. DOI:

10.1093/oxfordjournals.jhered.a111573

Shi Z, Lee JH, Oh DY, Yeo JS. 2010. Analysis of genetic and distances in
Asian cattle breeds using microsatellite markers. J. Korean Data Inf. Sci. Soc., 21: 795-802. DOI: http://www.dbpia.co.kr/Article/NODE07 244494

Tamura K, Stecher G, Peterson D, Filipski A, Kumar S. 2013. MEGA6: Molecular Evolutionary Genetics Analysis Version 6.0. Mol. Biol. Evol., 30: 2725-2729. 2013. DOI: $10.1093 / \mathrm{molbev} / \mathrm{mst} 197$

Thevenon S, Dayo GK, Sylla S, Sidibe I, Berthier D, Legros H, Boichard D, Eggen A, Gautier M. 2007. The extent of linkage disequilibrium in a large cattle population of western Africa and its consequences for association studies. Animal Genetics, 38: 277-286. DOI: 10.1111/j.1365-2052.2007.01601.x

Vaiman D, Mercier D, Moazami-Goudarzi K. 1994. A set of 99 cattle microsatellite characterization,synteny mapping and polymorphism. Mamm. Genome, 5: 2882897. DOI: $10.1007 / \mathrm{BF} 00389543$

Wright S. 1978. Evolution and the genetics of population. Variability Within and Among Natural Population, vol. 4. University of Chicago Press, Chicago, IL.

Ya-Bo Y, Jin-Yu W, Mekki DM, Qing-Ping T, Hui-Fang L, Rong G, Qing-Lian G, Wen-Qi Z, Kuanwei C 2006. Evaluation of genetic diversity and genetic distance between twelve Chinese indigenous chicken breeds based on microsatellite markers. Int. J. Poult. Sci., 5 : 550-556. DOI: $10.3923 /$ ijps.2006.550.556

Zangui MSI. 1986. L'élevage des bovins, ovins, caprins au Niger: Etude ethnologique. Thèse vèt., EISMV, Université de Dakar. 